FERMILAB-Conf-96/431

\title{
Discovering Technicolor at Hadron Colliders
}

\author{
John Womersley \\ Fermi National Accelerator Laboratory \\ P.O. Box 500, Batavia, Illinois 60510
}

December 1996

Presented at the 1996 DPF/DPB Summer Study on New Directions for High Energy Physics (Snowmass '96), Snowmass, Colorado, June 25-July 12, 1996 


\section{Disclaimer}

This report was prepared as an account of work sponsored by an agency of the United States Government. Neither the United States Government nor any agency thereof, nor any of their employees, makes any warranty, expressed or implied, or assumes any legal liability or responsibility for the accuracy, completeness, or usefulness of any information, apparatus, product, or process disclosed, or represents that its use would not infringe privately owned rights. Reference herein to any specific commercial product, process, or service by trade name, trademark, manufacturer, or otherwise, does not necessarily constitute or imply its endorsement, recommendation, or favoring by the United States Government or any agency thereof. The views and opinions of authors expressed herein do not necessarily state or reflect those of the United States Government or any agency thereof.

\section{Distribution}

Approved for public release; further dissemination unlimited. 


\title{
Discovering Technicolor at Hadron Colliders
}

\author{
John Womersley \\ Fermi National Accelerator Laboratory, Batavia, IL 60510*
}

\begin{abstract}
Strategies are presented for discovering light, color-singlet technipions $\left(\pi_{T}\right)$, produced in association with a vector boson through $s$-channel technirho production, at the Tevatron and LHC. Signal and $W+$ jets background were simulated including detector effects. Tagging of $b$-quarks from the $\pi_{T} \rightarrow b \bar{b}$ decay is found to be important to reduce the $W+$ jets background. The kinematic properties of signal and background events are significantly different and simple cuts can be used to further improve the signal to background ratio.
\end{abstract}

\section{INTRODUCTION}

Discovery strategies for light, color singlet technipions at hadron colliders have been investigated. As pointed out by Eichten and Lane[1] they can be copiously produced through $s$ channel technirho production:

$$
q \vec{q}^{\prime} \rightarrow \rho_{T}^{ \pm} \rightarrow V_{1} V_{2}
$$

where $V_{1} V_{2}=W^{ \pm} Z, W^{ \pm} \pi_{T}^{0}, \pi_{T}^{ \pm} Z$, or $\pi_{T}^{ \pm} \pi_{T}^{0}$; and through

$$
q \bar{q} \rightarrow \rho_{T}^{0} \rightarrow V_{1} V_{2}
$$

where $V_{1} V_{2}=W^{+} W^{-}, W^{ \pm} \pi_{T}^{\mp}$, or $\pi_{T}^{+} \pi_{T}^{-}$.

The modes where $W$ and $Z$ are produced and subsequently detected in their leptonic decays are straightforward and largely free of background; see for example the ATLAS Technical proposal[2]. In this study, the dijet decays of the technipion have been investigated:

$$
\begin{aligned}
& \pi_{T}^{0} \rightarrow b \bar{b} \\
& \pi_{T}^{ \pm} \rightarrow c \bar{b}
\end{aligned}
$$

These will generally be expected to dominate as long as the $t \bar{t}$ and $t \bar{b}$ modes are kinematically accessible; in Topcolor models the top decay modes can remain forbidden even for larger masses.

\section{SIGNAL AND BACKGROUND}

For definiteness the following process has been considered:

$$
q \bar{q}^{\prime} \rightarrow \rho_{T} \rightarrow W(\ell \nu) \pi_{T}(b \bar{b}),
$$

with $m_{\rho_{T}}=210 \mathrm{GeV}$ and $m_{\pi_{T}}=115 \mathrm{GeV}$. The signal is thus a $W$ (reconstructed from lepton plus missing transverse energy) together with two jets, with a resonance in the dijet mass $m_{j j}$. The backgrounds are $W+$ jets and $t \bar{t}$. (The latter has not yet been

\footnotetext{
* Operated by the Universities Research Association, Inc., for the U.S. Department of Energy
}

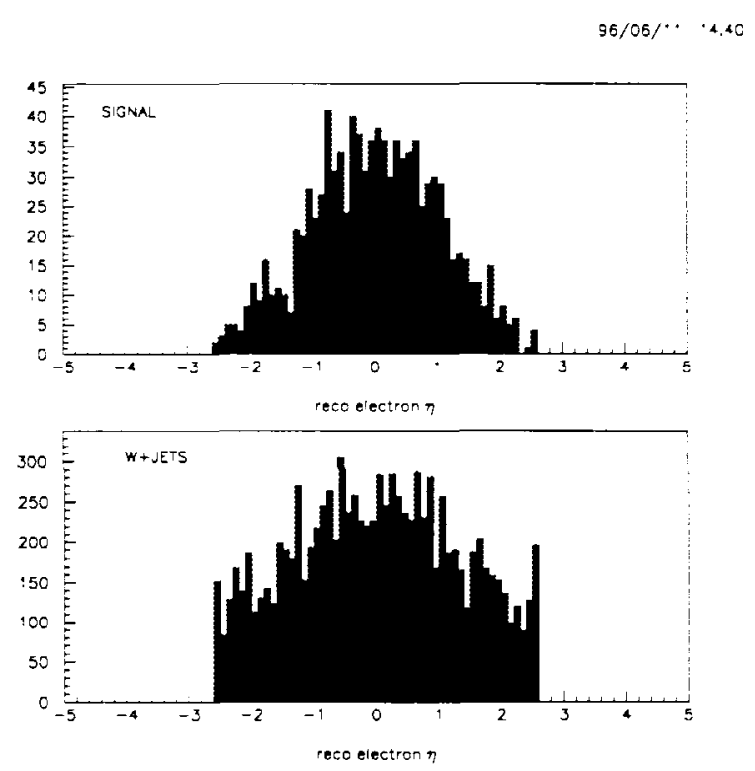

Figure 1: Reconstructed lepton pseudorapidity distributions for signal and $W+$ jets processes.

included in the study since it is small compared with the $W+$ jets process for $n=2$ jets, even if single $b$-tagging is applied). The signal cross sections are large: about $5 \mathrm{pb}$ at the Tevatron, and $35 \mathrm{pb}$ at the LHC [3]. The main issue is therefore dijet mass resolution and $b$-tagging.

Signal and background events were simulated using ISAJET. The signal topology was generated using the TCOLOR process with the $W Z$ final state; the $Z$ mass was set to $m_{\pi_{\tau}}$ and the decay to $b \bar{b}$ was forced.

Detector acceptance and resolution were modelled using a fast simulation[4]. Energy was deposited in cells of size $\Delta \eta \times$ $\Delta \phi=0.1 \times 0.1$ and was smeared for detector resolution: $15 \% / \sqrt{E(\mathrm{GeV})} \oplus 0.5 \%$ for $\mathrm{EM}$, and $50 \% / \sqrt{E(\mathrm{GeV})} \ominus 5 \%$ for hadronic energy. Transverse shower spreading and calorimeter leakage were also modeled. Jets were found (up to $|\eta|=4$ ) from the calorimeter towers using a cone of $R=0.7$. Missing transverse energy was calculated from the sum of the calorimeter towers over $|\eta| \leq 5$.

Events were selected which satisfied the following criteria:

- A good $W \rightarrow \ell \nu$ candidate, defined as:

- lepton with $p_{T}^{\ell}>25 \mathrm{GeV} / \mathrm{c},\left|\eta^{\ell}\right|<1.1$, and isolated (transverse energy within $R<0.4$ less than $10 \%$ of the lepton $p_{T}$ ); 


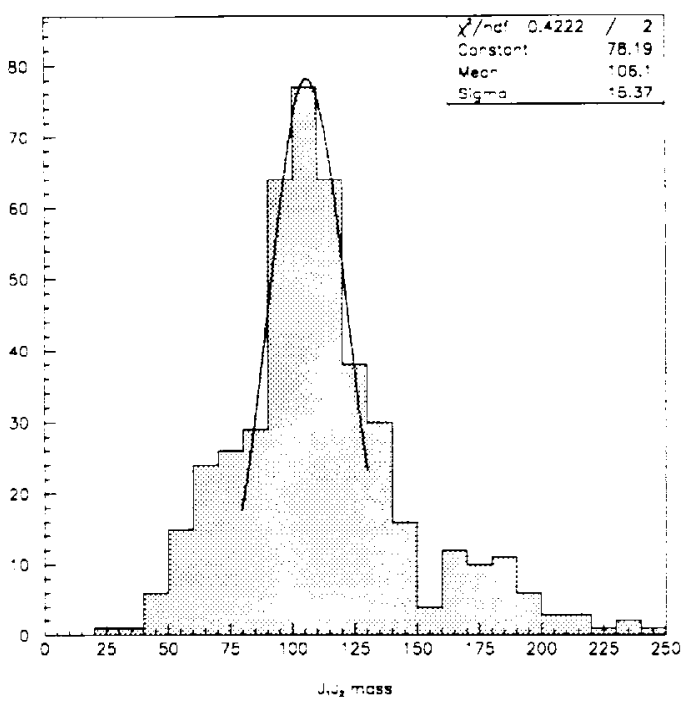

Figure 2: Leading dijet invariant mass distribution for $W(\ell \nu) \pi_{T}(b \bar{b})$ events at the LHC.

- $E_{T}^{\text {miss }}>25 \mathrm{GeV}$

- Transverse mass $m_{T}$ satisfying $50<m_{T}<$ $100 \mathrm{GeV}$;

- At least two jets with $E_{T}>20 \mathrm{GeV}$ and $\left|\eta^{j}\right|<2.5$.

The lepton was required to be central, since (as Fig. 1 shows) this gives some improvement in signal-to-background. For $b$ tagging it was assumed that single tagging would be performed with an efficiency of $50 \%$ and a mistag rate of $1 \%$ for light quark jets.

The cross sections obtained for the Tevatron and LHC are listed in Table I. It will be seen that the signal-to-background ratio for this process is rather better at the Tevatron.

Figure 2 shows the invariant mass distribution obtained for the leading jet pair in signal events. The peak has a resolution of about $15 \mathrm{GeV}$ with tails from jet combinatorics.

Table I: Cross sections for signal and $W+$ jets background.

\begin{tabular}{lcc}
\hline \hline & $W \pi_{T}$ & $W+$ jets \\
\hline LHC & & \\
\hline$\sigma \cdot B$ & $3.7 \mathrm{pb}$ & $2200 \mathrm{pb}$ \\
with 2 jets & $1.7 \mathrm{pb}$ & $250 \mathrm{pb}$ \\
with $b$-tag & $0.85 \mathrm{pb}$ & $2.5 \mathrm{pb}$ \\
\hline Tevatron & & \\
\hline$\sigma \cdot B$ & $0.53 \mathrm{pb}$ & $170 \mathrm{pb}$ \\
with 2 jets & $0.10 \mathrm{pb}$ & $2.5 \mathrm{pb}$ \\
with $b$-tag & $0.05 \mathrm{pb}$ & $0.025 \mathrm{pb}$ \\
\hline \hline
\end{tabular}

Figure 3 shows the invariant mass distribution obtained for

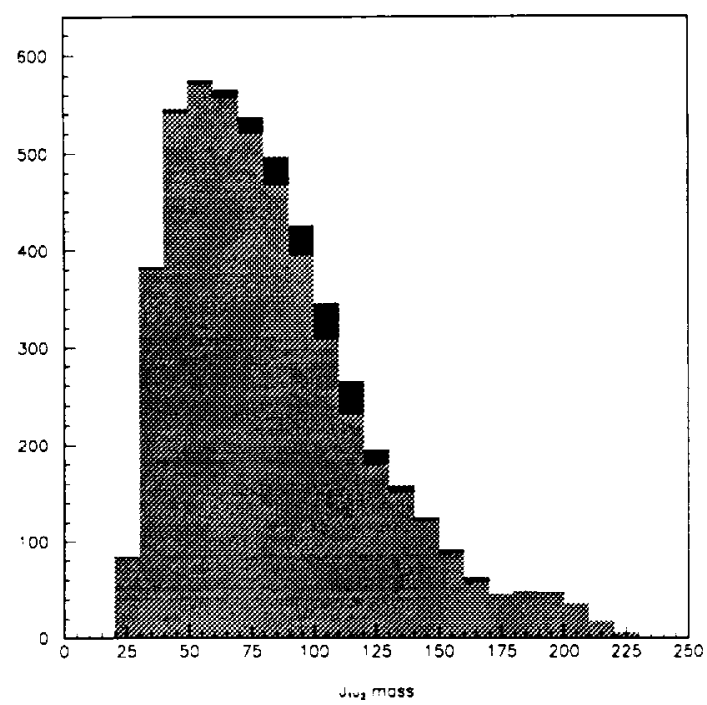

Figure 3: Leading dijet invariant mass distribution for technipion signal (dark) over the $W+$ jets background (light) at the Tevatron, before $b$-tagging. Vertical scale is events $/ 10 \mathrm{GeV} / 2 \mathrm{fb}^{-1}$. The background has been smoothed to simulate the full statistics.

signal and background at the Tevatron, before $b$-tagging. Once $b$-tagging is applied, the signal becomes much more apparent, as seen in Fig. 4. A clear excess is visible with $S: B \sim 5$ in the peak, and could easily be discovered in Run II with $2 \mathrm{fb}^{-} 1$. For comparison, Fig. 5 shows the situation at the LHC after $b$ tagging.

\section{KINEMATIC PROPERTIES OF THE EVENTS}

We note that there are significant differences in kinematic distributions between signal and background events. Cuts on these distributions may be used to further improve the background rejection, or they may be used as a way to confirm the presence of a signal by (for example) observing differences in these variables as a function of dijet mass.

Some variables of interest are:

- Transverse momentum of the leading dijet system, $p_{T}^{j j}$;

- Pseudorapidity of the leading dijet system, $\eta^{j j}$;

- $\Delta \phi$ between the leading two jets;

- Dijet asymmetry $A=\left(E_{T 1}-E_{T 2}\right) /\left(E_{T 1}+E_{T 2}\right)$.

The dijet pseudorapidity and asymmetry do not offer much discriminating potential, but the $\Delta \phi$ and transverse momentum of the dijet system are distinctly different between signal and background, as can be seen from Fig.6. Requiring, for example, 
$96 / \cdot 0 / 08 \cdots .04$

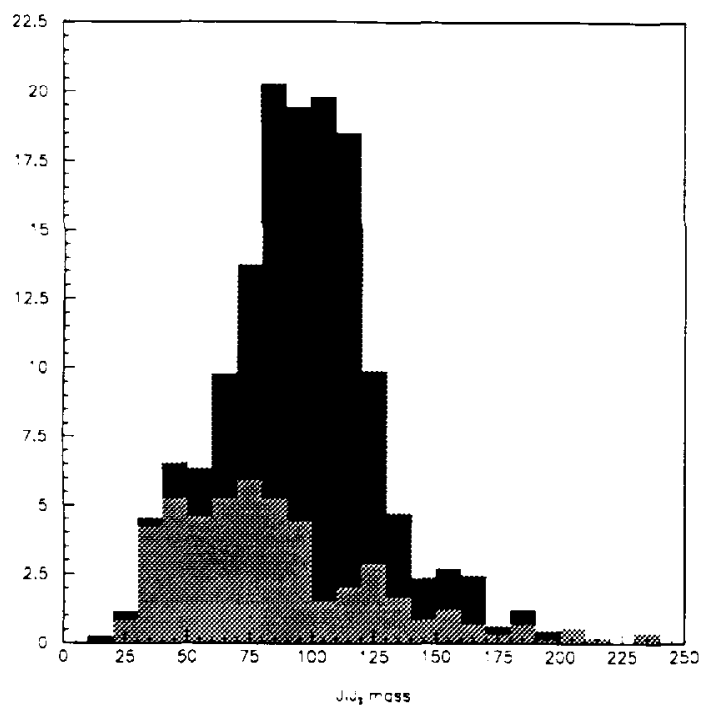

Figure 4: Leading dijet invariant mass distribution for technipion signal (dark) over the $W+$ jets background (light) at the Tevatron, after $b$-tagging. Vertical scale is events $/ 10 \mathrm{GeV} / 2 \mathrm{fb}^{-1}$.

$\Delta \phi>2.3$, and $p_{T}^{j j}<45 \mathrm{GeV} / \mathrm{c}$ retains $60 \%$ of the signal while rejecting $74 \%$ of the $W+$ jets background.

\section{CONCLUSIONS}

Light, color-singlet technipions, produced in association with a vector boson through s-channel technirho production, can be discovered at hadron colliders in the $b \bar{b}$ decay mode. The signal to background ratio is somehat better at the Tevatron but this physics can also be addressed at the LHC. Tagging of $b$-quarks is important to reduce the $W$ +jets background. The kinematic properties of signal and background events are significantly different and simple cuts can be used to further improve the signal to background ratio.

\section{REFERENCES}

[1] E. Eichten and K. Lane, 'Low-Scale Technicolor at the Tevatron', FERMILAB-PUB-96/075-T, hep-ph/9607213, and references therein.

[2] ATLAS Collaboration, Technical Proposal, CERN/LHCC/94-43, December 1994.

[3] K. Lane, 'Electroweak and Flavor Dynamics at Hadron Colliders', BUHEP-96-8, hep-ph/9605257

[4] A. Beretvas et al., 'SSCSIM: Development and Use by the Fermilab SDC group,' in Proc. of MC93, Intemational Conference on Monte Carlo Simulations in High Energy and Nuclear Physics, Tallahassee, FL, February 1993.

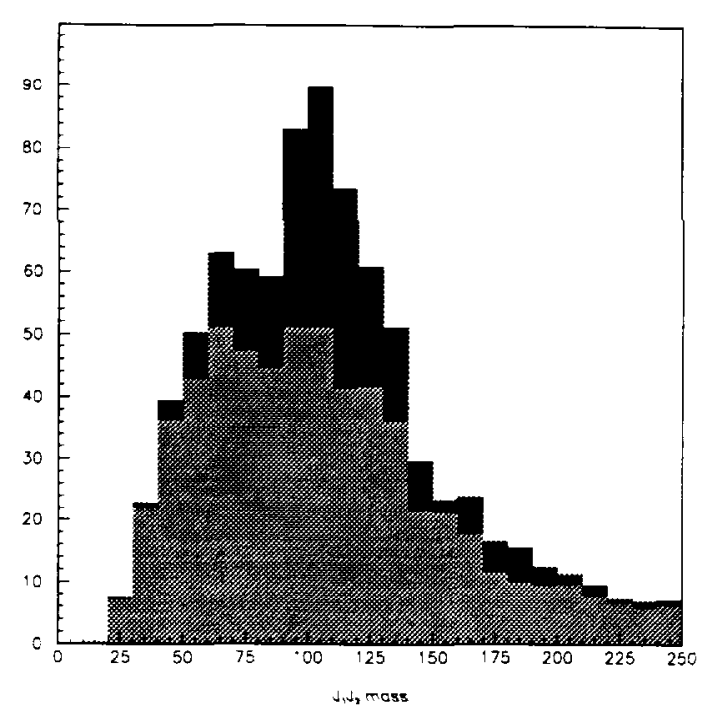

Figure 5: Leading dijet invariant mass distribution for technipion signal (dark) over the $W+$ jets background (light) at the LHC, after $b$-tagging. Vertical scale is events $/ 10 \mathrm{GeV} / 0.5 \mathrm{fb}^{-1}$.
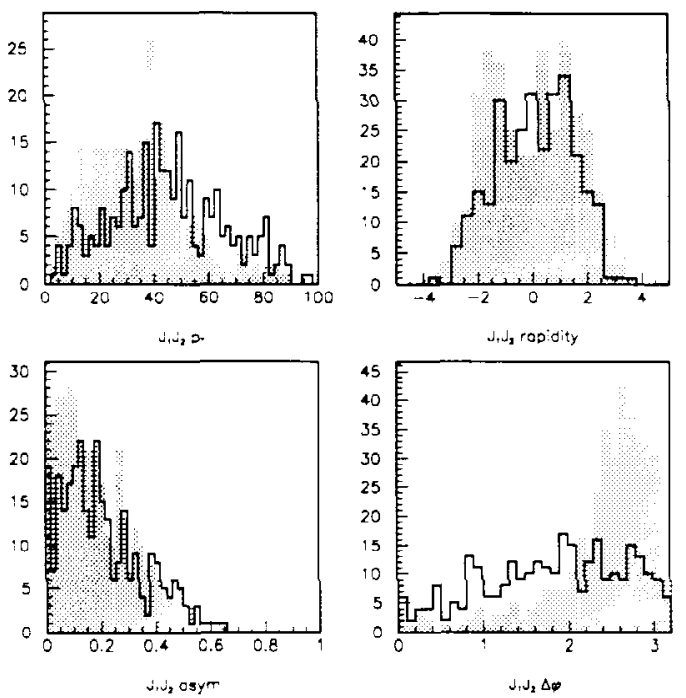

Figure 6: Distributions of $p_{T}^{j j}, \eta^{j j}$, asymmetry $A$ and $\Delta \phi$ for technipion signal (shaded) and $W+$ jets background (outline) at the Tevatron. 